Supporting Information for:

\title{
Lyotropic Liquid Crystal-based Membranes for Water Remediation: Fabrication, Characterization and Performance Evaluation
}

\author{
Liz Santiago-Martoral $^{1,2}$, Adrialis Figueroa ${ }^{1,3}$, and Eduardo Nicolau ${ }^{1,2^{*}}$ \\ ${ }^{1}$ Department of Chemistry, University of Puerto Rico, Rio Piedras Campus, 17 Ave. Universidad Ste. \\ 1701, San Juan, Puerto Rico USA 00925-2537 \\ ${ }^{2}$ Molecular Sciences Research Center, University of Puerto Rico, 1390 Ponce De Leon Ave, Suite 2, San \\ Juan Puerto Rico USA 00931-3346 \\ Corresponding Author \\ *Email: eduardo.nicolau@upr.edu
}



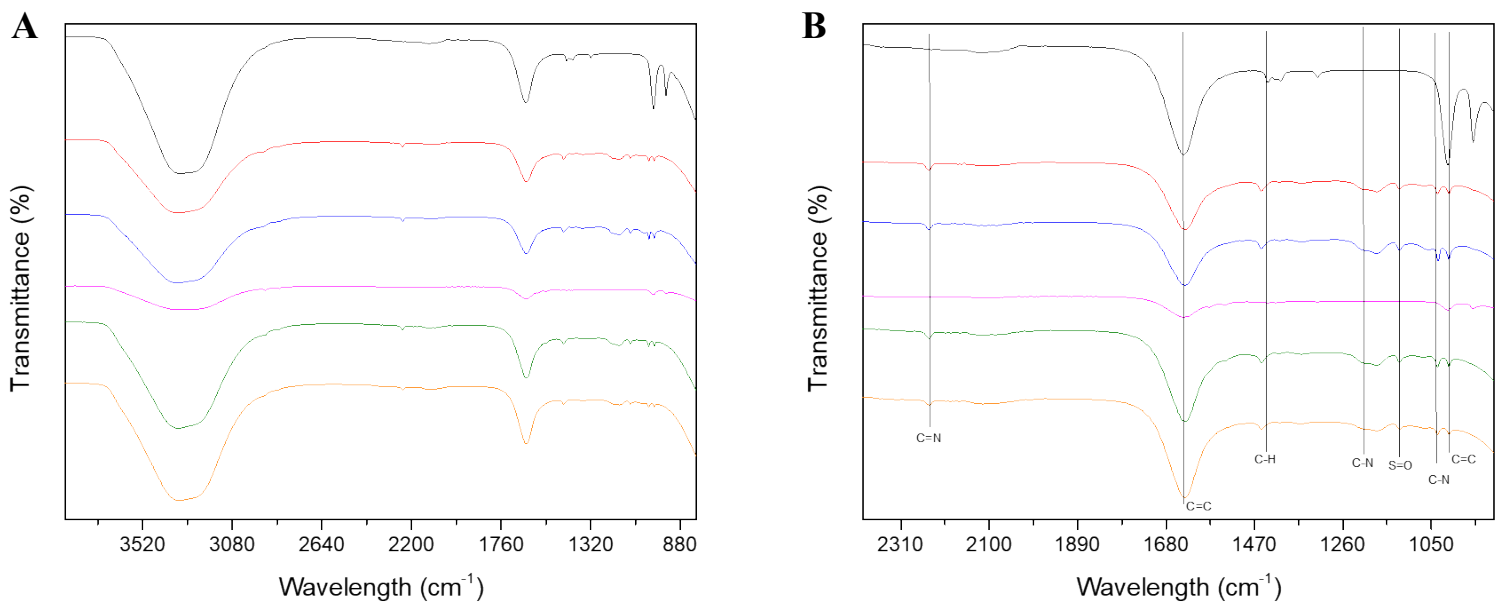

Figure S1. FTIR of membranes in all steps of preparation a) full spectra b) close-up. Black-SupP $100 \mu \mathrm{m}$, redSupPAC $100 \mu \mathrm{m}$, blue-SupPACMoDS $100 \mu \mathrm{m}$, magenta -SupP $120 \mu \mathrm{m}$, green-SupPAC $120 \mu \mathrm{m}$, orangeSupPACMoDS $120 \mu \mathrm{m}$.

FTIR spectra important surface modifications, sulfonate bands appear at 1195-1168 $\mathrm{cm}^{-1}$, nitrile $(\mathrm{C} \equiv \mathrm{N})$ $2260-2222 \mathrm{~cm}^{-1}$, imine $16-1168 \mathrm{~cm}^{-1}$, 


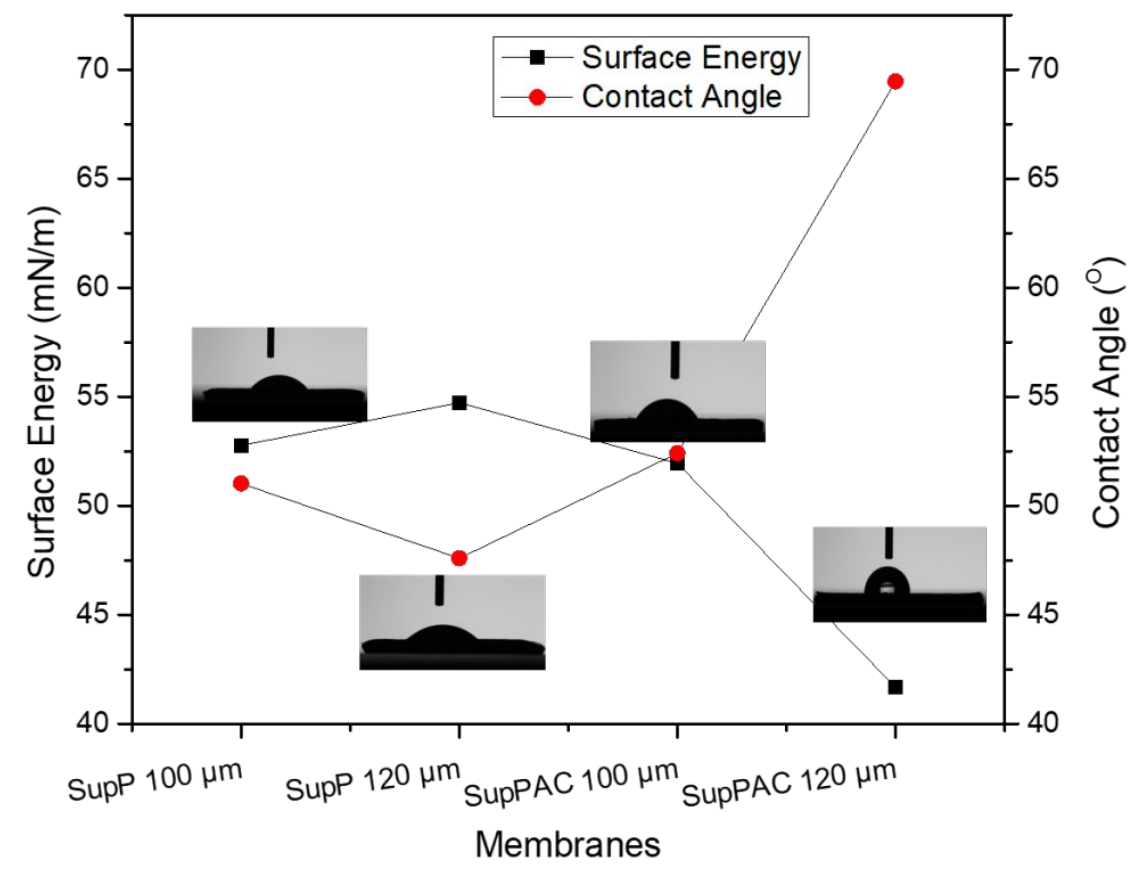

Figure S2. Contact Angle of the backside of the membranes. The SupP membranes are unmodified; therefore are slightly more hydrophilic than the SupPAC modified membranes, which are more hydrophobic. The higher porosity of this side of the membranes.

The membranes that did not have their active side modified with the LLC, i.e., SupP 100 and 120, present much lower contact angles than the active side of the membranes. For the reverse side of modified SupPAC 100 and 120 membranes, contact angles are considerably higher. On the reverse side, the SupPAC and SupPACMoDS have the same chemical composition. 